\title{
The orphan nuclear receptor Nur77 inhibits low shear stress-induced carotid artery remodeling in mice
}

\author{
YING YU $^{1 *}$, ZHAOHUA CAI $^{1 *}$, MINGLI CUI $^{1}$, PENG NIE $^{1}$, ZHE SUN $^{1}$, SHIQUN SUN $^{1}$, SHICHUN CHU $^{1}$, \\ XIAOLEI WANG ${ }^{1}$, LIUHUA HU ${ }^{1}$, JING YI ${ }^{2}$, LINGHONG SHEN ${ }^{1}$ and BEN HE ${ }^{1}$ \\ ${ }^{1}$ Department of Cardiology, Renji Hospital, School of Medicine, Shanghai Jiao Tong University, Shanghai 200127; \\ ${ }^{2}$ Department of Cell Biology, Key Laboratory of the Education Ministry for Cell Differentiation and Apoptosis, \\ Institutes of Medical Sciences, School of Medicine, Shanghai Jiao Tong University, Shanghai 200025, P.R. China
}

Received May 4, 2015; Accepted October 5, 2015

DOI: $10.3892 /$ ijmm.2015.2375

\begin{abstract}
Shear stress, particularly low and oscillatory shear stress, plays a critical pathophysiological role in vascular remodeling-related cardiovascular diseases. Growing evidence suggests that the orphan nuclear receptor Nur77 [also known as TR3 or nuclear receptor subfamily 4, group A, member 1 (NR4A1)] is expressed in diseased human vascular tissue and plays an important role in vascular physiology and pathology. In the present study, we used a mouse model of flow-dependent remodeling by partial ligation of the left common carotid artery (LCCA) to define the exact role of Nur77 in vascular remodeling induced by low shear stress. Following vascular remodeling, Nur77 was highly expressed in neointimal vascular smooth muscle cells (VSMCs) in the ligated carotid arteries. The reactive oxygen species (ROS) levels were elevated in the remodeled arteries in vivo and in primary rat VSMCs in vitro following stimulation with platelet-derived growth factor (PDGF). Further in vitro experiments revealed that Nur77 expression was rapidly increased in the VSMCs following stimulation with PDGF and $\mathrm{H}_{2} \mathrm{O}_{2}$, whereas treatment with $\mathrm{N}$-acetyl cysteine (NAC, a ROS scavenger) reversed the increase in the protein level of Nur77 induced by $\mathrm{H}_{2} \mathrm{O}_{2}$. Moreover, Nur77 overexpression markedly inhibited the proliferation and migration of VSMCs, induced by PDGF. Finally, to determine the in vivo role of Nur77 in low shear stress-induced vascular remodeling, wild-type (WT) and Nur77-deficient mice were subjected to partial ligation of the LCCA. Four weeks following surgery, in the LCCAs of the Nur77-deficient mice, a significant increase in the intima-media area and carotid intima-media thickness was noted, as well as
\end{abstract}

Correspondence to: Professor Ben He or Professor Linghong Shen, Department of Cardiology, Renji Hospital, School of Medicine, Shanghai Jiao Tong University, 1630 Dongfang Road, Shanghai 200127, PR. China

E-mail: rjheben@126.com

E-mail: rjshenlinghong@126.com

*Contributed equally

Key words: vascular remodeling, nuclear receptor, Nur77, reactive oxygen species, vascular smooth muscle cells more severe elastin disruption and collagen deposition compared to the WT mice. Immunofluorescence staining revealed an increase in VSMC proliferation [determined by the expression of proliferating cell nuclear antigen (PCNA)] and matrix metalloproteinase 9 (MMP-9) production in the Nur77-deficient mice. There was no difference in the number of intimal apoptotic cells between the groups. Taken together, our results indicate that Nur77 may be a sensor of oxidative stress and an inhibitor of vascular remodeling induced by low shear stress. Nur77, as well as its downstream cell signals, may thus be a potential therapeutic target for the suppression of vascular remodeling.

\section{Introduction}

Vascular remodeling is a complex pathophysiological process which is implicated in a number of cardiovascular diseases, such as atherosclerosis, hypertension, and restenosis following angioplasty. There is considerable evidence indicating that shear stress, particularly low and oscillatory shear stress, plays a critical role in vascular remodeling. For example, clinical and pathological data have revealed that atherosclerotic lesions occur preferentially at bifurcations and branch points, suggesting that low and disturbed shear stress correlate with vascular remodeling and atherogenesis (1). Even in healthy subjects, carotid intima-media thickness (IMT), a surrogate marker for early atherosclerosis, has been linked to low shear stress in the common carotid artery (2). In addition, shear stress is also responsible for the vascular remodeling associated with restenosis following angioplasty $(3,4)$.

Vascular remodeling induced by low shear stress involves multiple cell types, processes, various genes and signaling pathways. Of these, the excessive proliferation and migration of vascular smooth muscle cells (VSMCs) are the main pathological events leading to neointima formation and lumen narrowing. It is well known that platelet-derived growth factor (PDGF) is the major driving factor promoting abnormal VSMC proliferation and migration involved in vascular remodeling (5). Additionally, low shear stress promotes the production of reactive oxygen species (ROS) in a direct or indirect manner, and ROS can function as an intracellular second messenger to regulate many downstream signaling pathways, including those modulating the proliferation and migration of VSMCs $(6,7)$. Therefore, identi- 
fying new genes and signaling pathways that can indicate changes in wall shear stress or ROS levels and subsequently control the development of vascular remodeling is of great interest.

Nuclear receptors are a superfamily of transcription factors that are involved in diverse pathological functions. Several members of this superfamily play pivotal roles in atherosclerosis and vascular remodeling and have emerged as potential therapeutic targets for the treatment of cardiovascular diseases (8-10). The orphan nuclear receptor, Nur77 [also known as TR3 or nuclear receptor subfamily 4, group A, member 1 (NR4A1)], is an immediate early gene that plays a crucial role in the functional regulation of cell differentiation, proliferation, apoptosis, and inflammation. Previously, the biological effects of Nur77 in cardiovascular diseases, including atherosclerosis, vascular remodeling, cardiac ischemia/reperfusion injury and cardiac hypertrophy, have gained considerable attention (11-13). In 2000, Nur77 was first identified as one of 40 smooth muscle activationspecific genes (smags) in activated VSMCs, and it was noted that it is expressed in atherosclerotic lesions (14). Moreover, it has also been noted that Nur77 inhibits VSMC proliferation and attenuates neointima formation (15). Consistent with this previous study, more recent research from the same group indicated that Nur77 inhibits outward remodeling in SMC-specific overexpression of Nur77 transgenic mice (16). However, the direct role of Nur77 in the PDGF-induced VSMC proliferation and migration and vascular remodeling induced by low shear stress has not been extensively investigated as of yet.

In this study, we used a mouse model of vascular remodeling, which was induced by partial ligation of the left common carotid artery (LCCA). To the best of our knowledge, this is the first study that used Nur77-deficient mice to define the precise role of Nur77 in vascular remodeling induced by low shear stress. Our results suggest that oxidative stress is an important trigger for the upregulation of Nur77 during vascular remodeling, which in turn suppresses the proliferation and migration of VSMCs and the development of vascular remodeling.

\section{Materials and methods}

Mice. All animal experiments were performed according to the Shanghai Jiao Tong University School of Medicine (Shanghai, China) guidelines for the ethical care of animals. The protocol was approved by the Committee on the Ethics of Animal Experiments of the Shanghai Jiao Tong University School of Medicine [Permit no. (2013)-75]. Wild-type (WT) and Nur77 knockout (Nur77-KO) mice with a C57BL/6 background were purchased from Jackson Laboratory, (Bar Harbor, ME, USA) and were bred at Shanghai Biomodel Organism Science \& Technology Development Co., Ltd. Male WT and Nur77-KO mice (6-8 weeks of age, weighing 20-25 g) were used in this study and were randomly assigned to 2 groups $(n=8$ per group): partial ligation of the LCCA and the shamoperated control. All surgical procedures were performed under a dissecting microscope. All animals were sacrificed 4 weeks after the procedure.

Animal surgery. Partial ligation of the LCCA induces low shear stress in the LCCA and was performed as described previously (17). In brief, the mice were anesthetized with an intraperitoneal injection of pentobarbital sodium $(50 \mathrm{mg} / \mathrm{kg})$, and body temperature was maintained at $37^{\circ} \mathrm{C}$ using heating pads. After blunt dissection to expose the distal branches of the LCCA, blood flow was reduced by ligating all branches of the LCCA apart from the left thyroid artery. After ensuring that blood flow was present, the incision was closed with a suture. Sham ligation involved placing sutures, but no ligation was undertaken.

Tissue collection and processing. The mice were perfused with ice-cold isotonic saline, after which some left carotid arteries were isolated and stored at $-80^{\circ} \mathrm{C}$ until use. Others were fixed with $4 \%$ paraformaldehyde overnight, and were then embedded in optimal cutting temperature compound (OCT). OCT-embedded sections ( $8-\mu \mathrm{m}$-thick) were cut for use every $200 \mu \mathrm{m}$ over a $2 \mathrm{~mm}$ length of carotid artery, from the distal bifurcation of carotid artery specimens.

Histological staining. The OCT-embedded sections were stained with hematoxylin and eosin (H\&E; Sigma, St. Louis, MO, USA) using a standard protocol. Picrosirius red (Sigma) and Verhoeff-Van Gieson (Genmed Scientifics, Arlington, VA, USA) stains were used for staining collagen and elastin, respectively. For staining collagen, the sections were fixed with $4 \%$ paraformaldehyde for $20 \mathrm{~min}$ and then washed with double-distilled water 3 times for $5 \mathrm{~min}$. The sections were then immersed in $0.1 \%$ picric acid (Sigma) solution and dehydrated using absolute ethyl alcohol (Zhenxing Chemical, Shanghai, China) after being washed twice with $0.5 \%$ acetic acid. Elastin was determined according to the manufacturer's instructions provided with the Elastin Stain kit (Genmed Scientifics). All images were captured using an Olympus digital camera (Olympus, Tokyo, Japan) and analyzed using ImagePro Plus software.

Immunofluorescence staining. OCT-embedded sections were washed with phosphate-buffered saline (PBS) and then fixed in $4 \%$ paraformaldehyde for $20 \mathrm{~min}$, after which time the sections were permeabilized with $0.2 \%$ Triton X-100 (Sigma) for $8 \mathrm{~min}$. The sections were blocked with $5 \%$ fetal bovine serum (FBS; Gibco, Grand Island, NY, USA) for $30 \mathrm{~min}$ and incubated with primary antibodies against Nur77 (1:100; \#ab13851; Abcam, Cambridge, UK), smooth muscle (SM)- $\alpha$-actin (1:300; \#ab21027; Abcam), matrix metalloproteinase-9 (MMP-9) (1:100; \#ab38898; Abcam) and proliferating cell nuclear antigen (PCNA) (1:100; \#AJ1594a; Abgent, San Diego, CA, USA) overnight, followed by further staining with secondary antibodies labeled with red fluorescence (1:300;\#A31570; donkey-anti-mouse; $555 \mathrm{~nm}$ ) and green fluorescence (1:300; \#A21206; donkey-anti-rabbit; $488 \mathrm{~nm}$ ) (both from Invitrogen, Carlsbad, CA, USA) for $60 \mathrm{~min}$. After staining the nuclei with 4,6-diamidino-2-phenylindole (DAPI; blue fluorescence; Beyotime, Shanghai, China), the fluorescence signal was acquired using a confocal microscope (Zeiss LSM 710; Carl Zeiss, Oberkochen, Germany).

Terminal deoxynucleotidyltransferase-mediated dUTP nick-end labelling (TUNEL) assay. TUNEL staining was performed using the In Situ Cell Death Detection kit (Roche Diagnostics, Madison, WI, USA). Briefly, the LCCA segments were fixed in $4 \%$ paraformaldehyde for $20 \mathrm{~min}$ and permeabilized with $0.1 \%$ Triton X-100 and $0.1 \%$ sodium citrate in PBS on ice for $2 \mathrm{~min}$. Tissue samples were then incubated with TUNEL reagent at $37^{\circ} \mathrm{C}$ for $50 \mathrm{~min}$. Following incubation, the 
nuclei were stained with DAPI for another 8 min. Images were acquired using a confocal microscope (Zeiss LSM 710).

Detection of ROS. 2',7'-Dichlorodihydrofluorescein diacetate (DCFH-DA; Sigma) was used to measure the ROS levels in the cultured VSMCs, as previously described (18). Dihydroethidium (DHE; Sigma) staining was used for the in situ detection of ROS levels in frozen tissue sections, which were mounted in OCT-embedding compound and frozen at $-20^{\circ} \mathrm{C}$. Briefly, unfixed frozen cross-sections were incubated with $\mathrm{DHE}(5 \mu \mathrm{mol} / \mathrm{l})$ at $37^{\circ} \mathrm{C}$ for $30 \mathrm{~min}$ in a humidified chamber protected from light, followed by a 5-min wash in PBS. Images were obtained by confocal microscopy (Zeiss LSM 710).

Cell culture. Primary VSMCs were isolated from the thoracic aortic arteries of Sprague-Dawley rats (6-8 weeks of age) using mechanical dissociation, as previously described (19). Briefly, the medial layer of the rat thoracic aorta was isolated surgically and minced into approximately $1-\mathrm{mm}^{2}$ pieces, which were plated into 6-cm dishes for culture in DMEM with $10 \% \mathrm{FBS}, 100 \mathrm{U} /$ $\mathrm{ml}$ penicillin, $100 \mu \mathrm{g} / \mathrm{ml}$ streptomycin, and incubated at $37^{\circ} \mathrm{C}$, $5 \% \mathrm{CO}_{2}$. VSMCs were characterized by immunofluorescence staining for SM- $\alpha$-actin. VSMCs at passages 3-8 were used in all experiments. Twenty-four hours prior to drug treatment, the VSMCs were transferred to serum-free medium for the duration of the experiment. The VSMCs were treated with the vehicle (solvent control) or various reagents, including PDGF (R\&D Systems, Minneapolis, $\mathrm{MN}, \mathrm{USA}$ ), $\mathrm{H}_{2} \mathrm{O}_{2}$ (Sigma) and the antioxidant, $\mathrm{N}$-acetyl cysteine (NAC; Sigma), as described in the figure legends.

Western blot analysis. Whole cell lysates were prepared and quantitated by Bradford assay. Equal amounts of protein per lane were subjected to SDS-PAGE, transferred onto a nitrocellulose membranes, and western blot analysis was performed using antibodies against Nur77 (1:1,000; \#ab109180; Abcam), $\beta$-actin (1:5,000; \#ab6276; Abcam), GFP (1:1,000; \#ab290; Abcam), PCNA (1:1,000; \#AJ1594a; Abgent), cyclin D1 (1:1,000; \#2978; Cell Signaling Technology, Danvers, MA, USA) and horseradish peroxidase-conjugated secondary antibodies (1:5,000; \#115-035-003/111-035-003, Jackson ImmunoResearch Laboratories, West Grove, PA, USA). Protein bands were detected using enhanced chemiluminescence (Millipore, Billerica, MA, USA), and quantification was performed using Quantity One 4.4.0 software (Bio-Rad, Hercules, CA, USA).

Reverse transcription-quantitative polymerase chain reaction $(R T-q P C R)$. Total RNA was extracted from the cells using TRIzol reagent (Invitrogen) according to the manufacturer's instructions. The purity of the total RNA was assessed by ultraviolet spectrophotometry. Total RNA $(2 \mu \mathrm{g})$ was reverse transcribed into cDNA. cDNA was then used as the template in a $20-\mu 1$ qPCR reaction, which was completed using the ABI Prism 7300 real-time PCR system (Applied Biosystems, Foster City, CA, USA). The primers used were as follows: $\beta$-actin forward, 5'-GGC ATC GTC ACC AAC TGG GAC-3' and reverse, 5'-CGA TTT CCC GCT CGG CCG TGG-3'; Nur77 forward, 5'-GCT CAT CTT CTG CTC AGG CCT-3' and reverse, 5'-CAG ACG TGA CAG GCA GCT GGC-3'.
Cell counts and cell counting kit-8 (CCK-8) assay. For cell counts, primary rat VSMCs were plated into a 24-well plate and were infected with green fluorescent protein (GFP)-Nur77 adenovirus (Ad-GFP-Nur77) and GFP control adenovirus (Ad-GFP) for $48 \mathrm{~h}$. The cells were subsequently stimulated with PDGF $(20 \mathrm{ng} / \mathrm{ml})$ for another $24 \mathrm{~h}$. The cells were then trypsinized and counted using a hemocytometer and an Olympus inverted microscope. For the CCK-8 assay, primary VSMCs were plated into a 96-well plate. The cells were infected with adenovirus and stimulated with PDGF as mentioned above. Cell proliferation was assessed by CCK-8 assay (Yeasen, Shanghai, China), according to the manufacturer's instructions.

In vitro scratch-wound assay. The primary rat VSMCs were cultured on 6-well plates and infected with Ad-GFP-Nur77 or Ad-GFP for $48 \mathrm{~h}$. Confluent cells were growth-arrested and scraped using sterilized $10-\mu l$ pipette tips, washed with PBS, and stimulated with PDGF ( $20 \mathrm{ng} / \mathrm{ml})$ for $24 \mathrm{~h}$. The cells were visualized on an Olympus inverted microscope.

Statistical analysis. Values are expressed as the means \pm standard error of the mean (SEM). Differences between groups were compared by two-tailed Student's t-tests. All tests were two-sided, and a value of $\mathrm{p}<0.05$ was considered to indicate a statistically significant difference.

\section{Results}

Nur77 is highly expressed in neointimal VSMCs in the ligated carotid arteries following vascular remodeling induced by low shear stress. Partial ligation of the LCCA was performed to induce low shear stress and to generate a model of carotid artery remodeling, as previously described (17). As shown in Fig. 1A, partial carotid ligation resulted in significant vascular remodeling, as assessed by H\&E staining and quantification of the intimamedia area $\left(20,324.65 \pm 1,143.16\right.$ vs. $32,793.74 \pm 1,940.77 \mu \mathrm{m}^{2}$, $\mathrm{p}<0.05)$ and $\operatorname{IMT}(12.35 \pm 1.29$ vs. $25.16 \pm 2.02 \mu \mathrm{m}, \mathrm{p}<0.05)$. Accordingly, in the ligated carotid arteries, we noted severe elastin disruption and more collagen deposition, as assessed by Verhoeff-Van Gieson staining and picrosirius red staining, respectively (Fig. 1B).

To determine whether Nur77 plays a role in the vascular remodeling induced by low shear stress, we examined the expression of Nur77 in neointimal VSMCs in the ligated carotid arteries by dual immunofluorescence staining with specific antibodies against Nur77 or SM- $\alpha$-actin. As shown in Fig. 1C, Nur77 was highly expressed in the neointimal VSMCs in the ligated carotid arteries following vascular remodeling compared to the VSMCs in the arteries from the sham-operated control. Moreover, Nur77 was localized predominantly in the neointimal VSMCs in the ligated carotid arteries.

Nur77 expression is upregulated by PDGF in a ROS-dependent manner in primary rat VSMCs. PDGF is known to be involved in vascular remodeling induced by low shear stress (20). Therefore, we examined the expression of Nur77 in rat VSMCs in response to PDGF stimulation. As shown in Fig. 2A, stimulation with PDGF $(20 \mathrm{ng} / \mathrm{ml})$ induced a rapid increase in Nur77 mRNA levels, peaking at $1 \mathrm{~h}$. Subsequently, the protein expression of Nur77 was examined by western blot analysis 
A
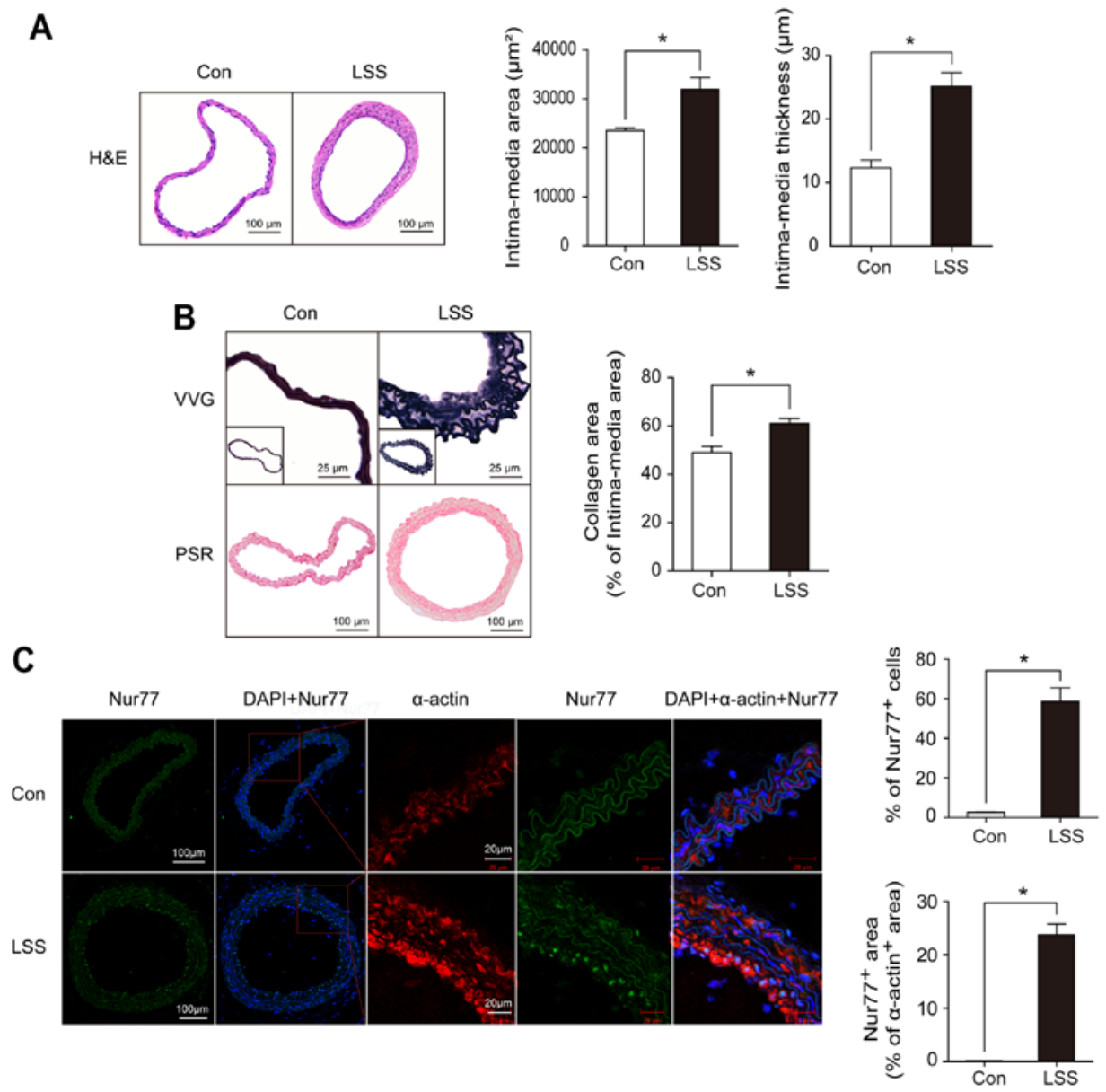

Figure 1. Expression of Nur77 in neointimal vascular smooth muscle cells (VSMCs) following vascular remodeling induced by low shear stress (LSS). (A) Representative images of $\mathrm{H} \& \mathrm{E}$ staining (scale bar, $100 \mu \mathrm{m}$ ) and quantification of the intima-media area and intima-media thickness in the control (Con) and LSS groups ( $(\mathrm{p}<0.05, \mathrm{n}=8$ ). (B) Representative images of Verhoeff-Van Gieson staining (VVG) and picrosirius red staining (PSR) (scale bar, $100 \mu \mathrm{m})$ and quantification of the collagen area ( $\left.{ }^{*} \mathrm{p}<0.05, \mathrm{n}=8\right)$. (C) Representative images of dual immunofluorescence staining of Nur77 and smooth muscle (SM)- $\alpha$-actin, and quantification of percentage of Nur77-positive cells and the ratio of Nur77-positive area to the $\alpha$-actin-positive area ( $\left.{ }^{*} \mathrm{p}<0.05, \mathrm{n}=6\right)$. Values represent the means \pm SEM.

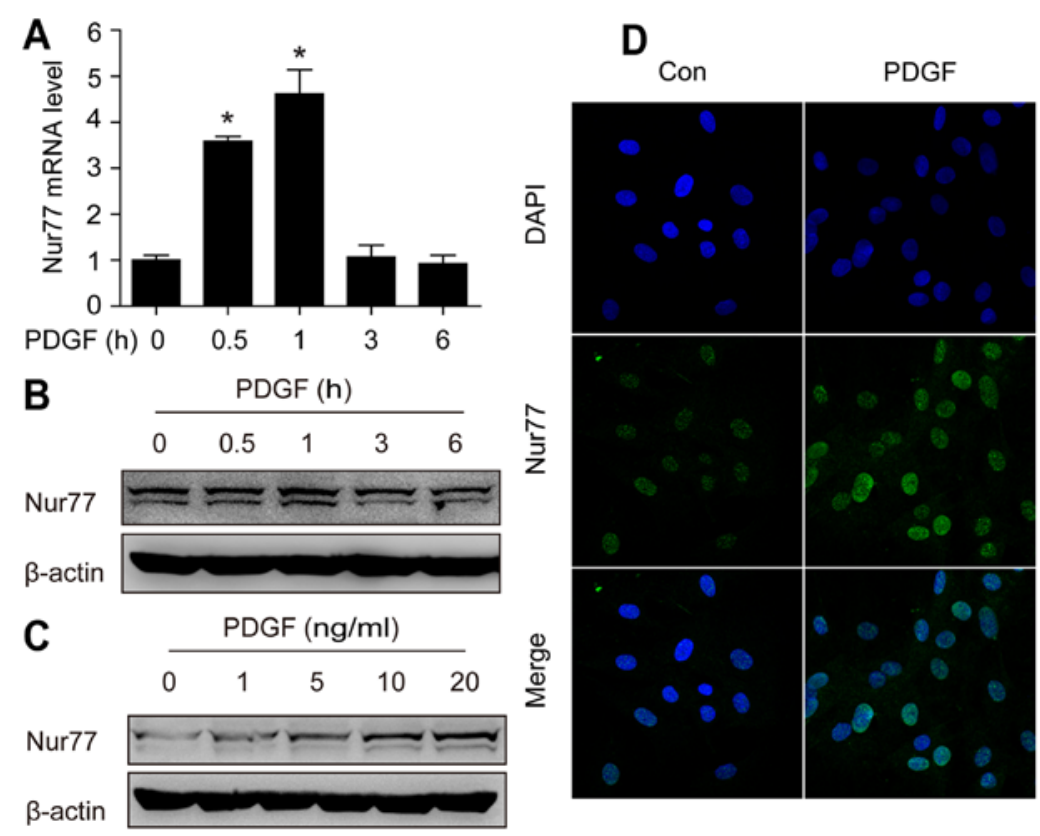

Figure 2. Platelet-derived growth factor (PDGF) induces Nur77 expression in primary rat vascular smooth muscle cells (VSMCs). (A and B) Primary rat VSMCs were serum-starved for $24 \mathrm{~h}$ and then stimulated with PDGF $(20 \mathrm{ng} / \mathrm{ml})$ for the indicated periods of time. (A) mRNA and (B) protein levels of Nur77 were quantified by RT-qPCR and western blot analysis, respectively ( $\mathrm{p}<0.05)$. (C) Primary rat VSMCs were treated with increasing concentrations of PDGF for $1 \mathrm{~h}$. Protein expression of Nur77 was examined by western blot analysis. (D) Representative confocal images of rat VSMCs treated with the vehicle or PDGF (20 ng/ml) for $1 \mathrm{~h}$ and stained for Nur77 (green) and nuclei (blue). Values represent the means \pm SEM. Con, control. 
A

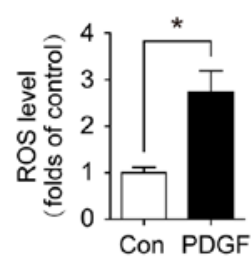

C

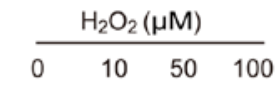

Nur77

B-actin
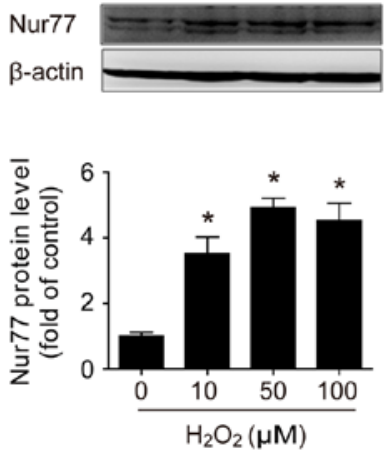

B
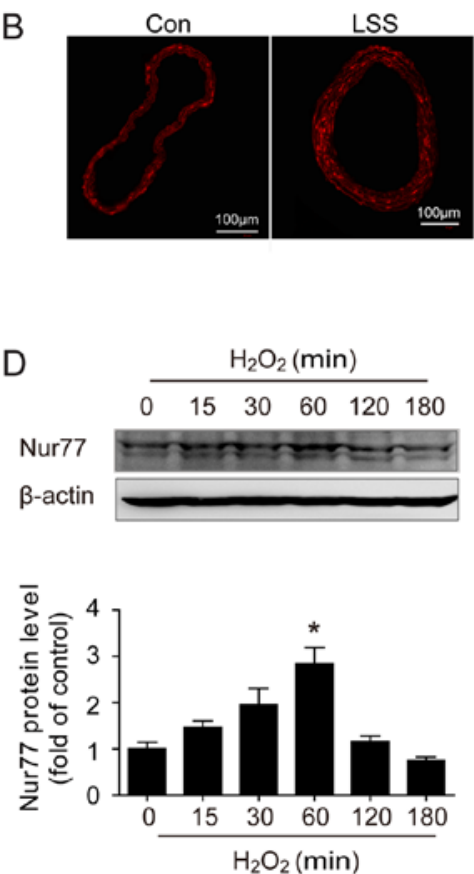
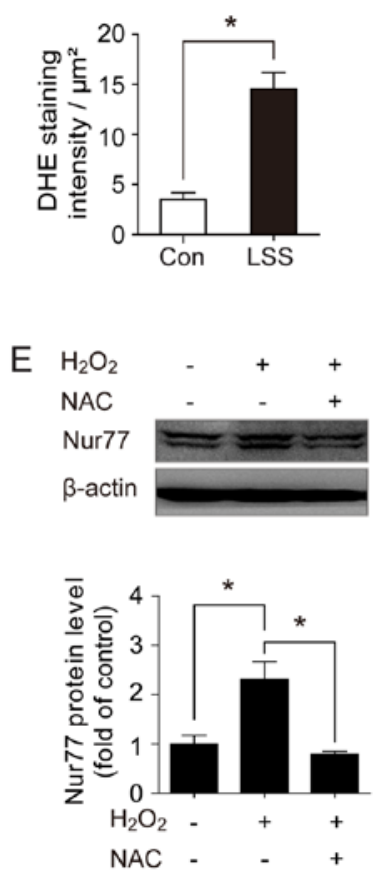

Figure 3. Upregulation of Nur77 is reactive oxygen species (ROS)-dependent in primary rat vascular smooth muscle cells (VSMCs). (A) Primary rat VSMCs were treated with platelet-derived growth factor (PDGF) $(20 \mathrm{ng} / \mathrm{ml})$ for $30 \mathrm{~min}$. ROS levels were detected by flow cytometry following DCFH-DA staining. (B) Representative images of dihydroethidium (DHE) staining (scale bar, $100 \mu \mathrm{m}$ ) and quantification of DHE staining intensity in control (Con) and low shear stress (LSS) groups ( ${ }^{*} \mathrm{p}<0.05, \mathrm{n}=6$ ). (C) Primary rat VSMCs were treated with increasing concentrations of $\mathrm{H}_{2} \mathrm{O}_{2}$ for $1 \mathrm{~h}$. And protein expression of Nur77 was examined by western blot analysis. (D) Primary rat VSMCs were stimulated with $\mathrm{H}_{2} \mathrm{O}_{2}(100 \mu \mathrm{M})$ for the indicated periods of time. Protein expression of Nur77 was examined by western blot analysis. (E) Primary rat VSMCs were treated with vehicle or $5 \mathrm{mM} \mathrm{N}$-acetyl cysteine ( $\mathrm{NAC}$ ) for $6 \mathrm{~h}$ prior to $\mathrm{H}_{2} \mathrm{O}_{2}$ stimulation for another $1 \mathrm{~h}$. Protein expression of Nur77 was examined by western blot analysis $\left({ }^{*} \mathrm{p}<0.05\right)$. Values represent the means \pm SEM.

and immunofluorescence staining. As shown in Fig. 2B and C, PDGF stimulation led to rapid increase in the protein expression of Nur77 in a dose-dependent manner. Immunofluorescence staining revealed similar results (Fig. 2D).

A growing body of evidence indicates that oxidative stress plays a critical pathophysiological role in vascular remodeling (21-24). Therefore, to determine whether Nur77 expression is mediated by oxidative stress, we examined whether Nur77 expression was induced following an induction of ROS production. As shown in Fig. 3A, stimulation with PDGF increased the intracellular ROS levels in vitro in the primary VSMCs. Similarly, the ROS levels were significantly increased in the ligated carotid arteries (Fig. 3B). Furthermore, we examined the expression of Nur77 in response to $\mathrm{H}_{2} \mathrm{O}_{2}$ stimulation. The results revealed that $\mathrm{H}_{2} \mathrm{O}_{2}$ stimulation induced an upregulation in Nur77 expression in a dose-dependent manner (Fig. 3C). An obvious increase in Nur77 protein expression was noted following 15 min of exposure to $\mathrm{H}_{2} \mathrm{O}_{2}$, and reached a plateau at $1 \mathrm{~h}$ (Fig. 3D). Moreover, the upregulation of Nur77 expression was blocked by treatment with the antioxidant, NAC (Fig. 3E), suggesting that the upregulation of Nur77 is ROS-dependent. Taken together, these results suggest that ROS may be the direct or indirect reason for the upregulation of Nur77 during vascular remodeling.

Nur77 inhibits the PDGF-induced proliferation and migration of VSMCs. To establish the functional significance of Nur77, we examined the effects of Nur77 overexpression on the PDGF-induced proliferation and migration of VSMCs. We infected the VSMCs with Ad-GFP-Nur77 or Ad-GFP (Fig. 4A). As shown in Fig. 4B and C, the Ad-GFP-Nur77-infected cells exhibited decreased proliferation following stimulation with PDGF compared with the Ad-GFP-infected cells. Consistent with this result, the levels of cyclin D1 and PCNA, which reflect the ability of VSMCs to proliferate, were markedly decreased in the Ad-GFP-Nur77-infected cells (Fig. 4D). Additionally, the in vitro scratch-wound assay revealed decreased VSMC migration in the Ad-GFP-Nur77-infected cells compared with the Ad-GFP-infected cells (Fig. 4E). These results suggest that Nur77 inhibits the proliferation and migration of VSMCs.

Nur77 deletion enhances vascular remodeling induced by low shear stress. In order to further define the in vivo role of Nur77 in vascular remodeling induced by low shear stress, partial carotid ligation was performed in age- and gender-matched WT and Nur77-deficient mice. Four weeks following surgery, the Nur77-deficient mice exhibited markedly enhanced vascular remodeling compared with the WT mice, as assessed by H\&E staining (Fig. 5A). Quantitative morphometric analysis revealed a marked increase in the intima-media area $(38,08$ $0 \pm 2,317$ vs. $\left.29,420 \pm 1,851 \mu \mathrm{m}^{2}, \mathrm{p}<0.05\right)$ and IMT $(52.01 \pm 3$ .27 vs. $29.54 \pm 2.76 \mu \mathrm{m}, \mathrm{p}<0.05)$ in the ligated carotid arteries from the Nur77-deficient mice compared to those from the WT mice (Fig. 5A). Verhoeff-Van Gieson staining revealed more severe elastin disruption following partial carotid ligation in the Nur77-deficient mice (Fig. 5B). Similarly, picrosirius red staining, indicating collagen deposition, was more pronounced in the ligated carotid arteries from the Nur77-deficient mice (Fig. 5B).

Dual immunofluorescence staining for PCNA and SM- $\alpha$-actin was performed in the ligated carotid arteries. The ratio of the PCNA-positive area to the SM- $\alpha$-actin-positive 
A
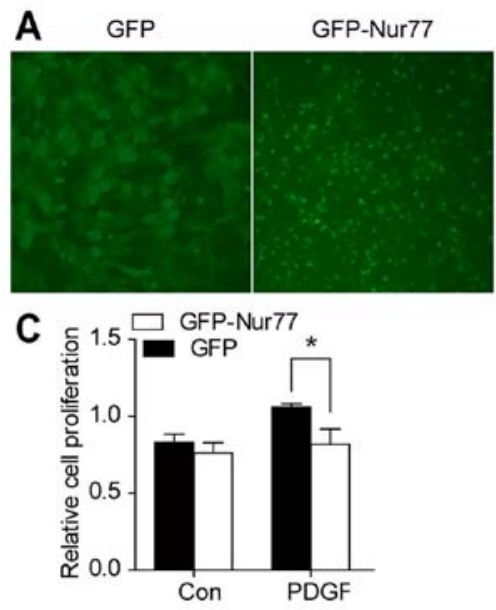

D

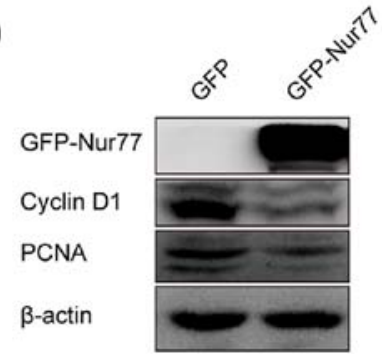

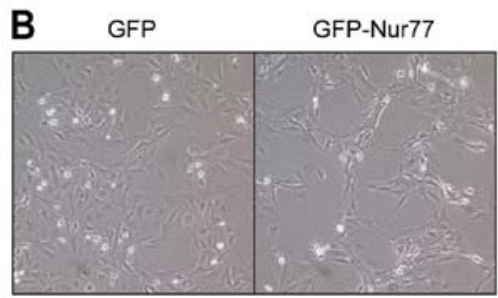

$\mathbf{E}$
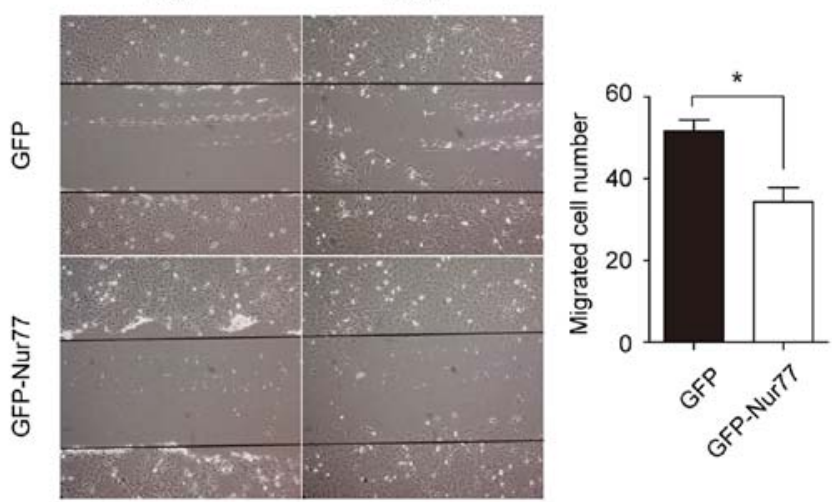

Figure 4. Nur77 inhibits platelet-derived growth factor (PDGF)-induced vascular smooth muscle cell (VSMC) proliferation and migration in primary VSMCs in vitro. (A) Representative images of green fluorescent protein (GFP)-Nur77 adenovirus (Ad-GFP-Nur77) and GFP control adenovirus (Ad-GFP) infection in VSMCs in vitro. (B and C) Representative images of (B) cell density and quantification, as measured by cell count and (C) cell counting kit-8 (CCK-8) assay in Ad-GFP-Nur77-infected cells compared with Ad-GFP-infected cells upon PDGF (20 ng/ml) stimulation ("p<0.05). (D) Western blot analysis measuring levels of GFP-Nur77, cyclin D1 and proliferating cell nuclear antigen (PCNA). $\beta$-actin was used as an internal control. (E) Representative images of the in vitro scratch-wound assay and quantification of number of migrated cells $\left({ }^{*} \mathrm{p}<0.05\right)$. Values represent the means $\pm \mathrm{SEM}$. Con, control.

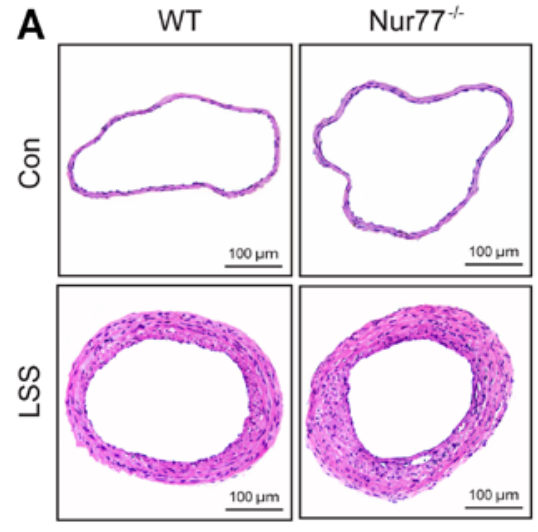

B

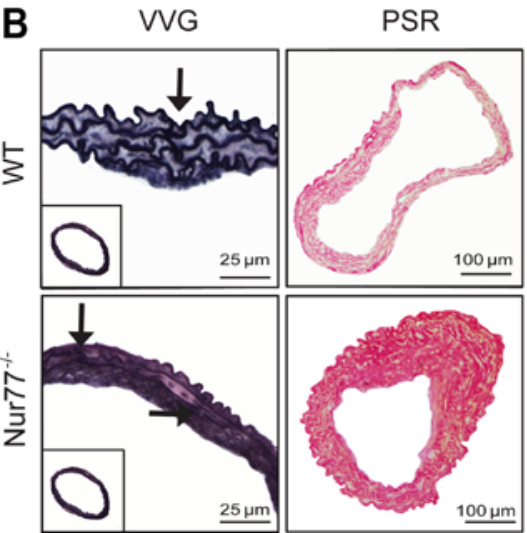

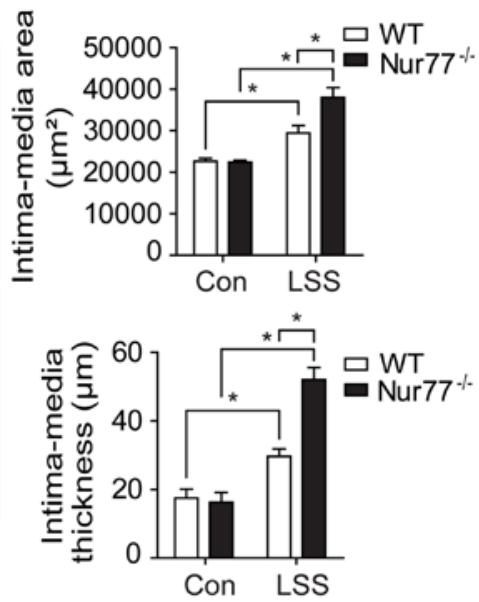

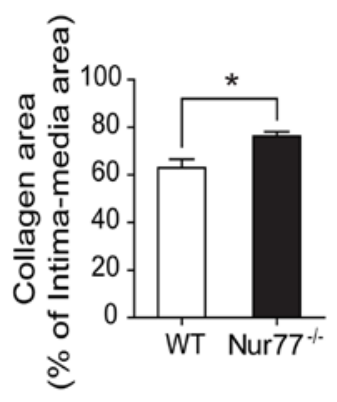

Figure 5. Nur77 deletion enhances vascular remodeling induced by low shear stress (LSS) in vivo. (A) Representative images of H\&E staining (scale bar, $100 \mu \mathrm{m}$ ) and quantification of the intima-media area and intima-media thickness in wild-type (WT) and Nur77-deficent mice ( $\mathrm{p}<0.05$, $\mathrm{n}=8)$. (B) Representative images of Verhoeff-Van Gieson (VVG) staining (scale bar, $25 \mu \mathrm{m}$ ), picrosirius red staining (PSR) (scale bar, $100 \mu \mathrm{m}$ ), and quantification of collagen area in WT and Nur77-deficent mice ( $(\mathrm{p}<0.05, \mathrm{n}=8)$. Arrows point to disrupted elastin structure. Values represent the means \pm SEM.Con, control. 

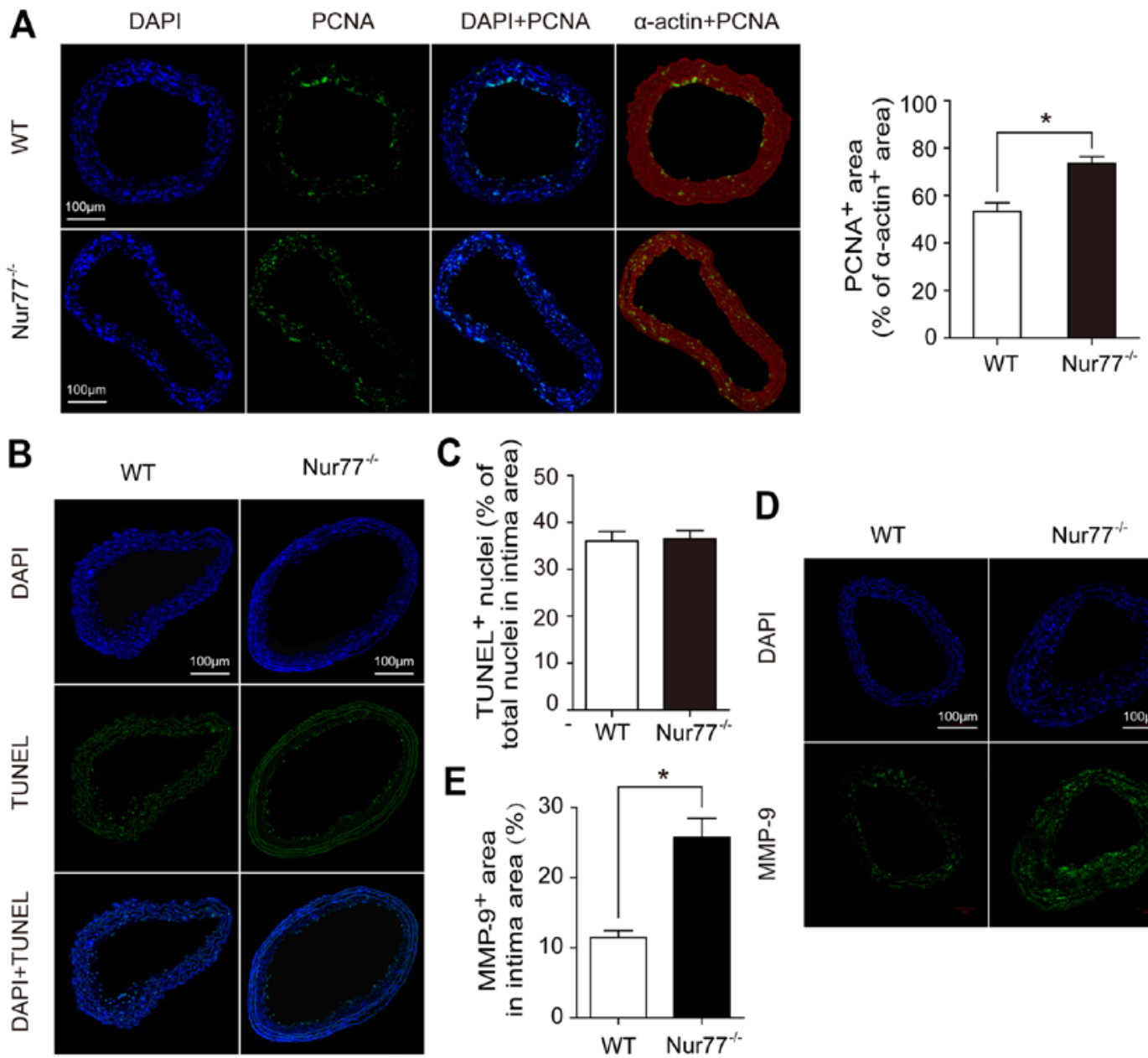

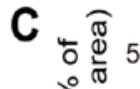

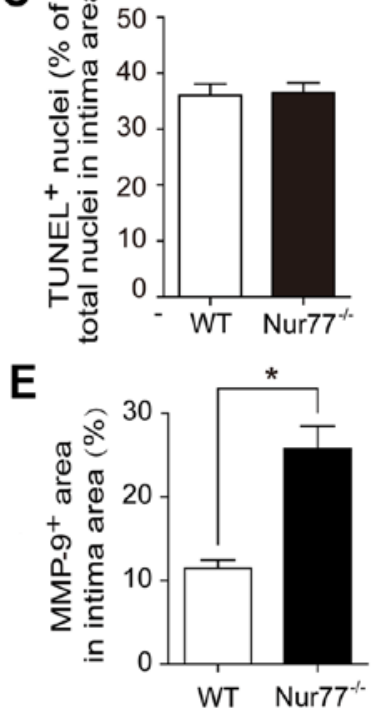

D

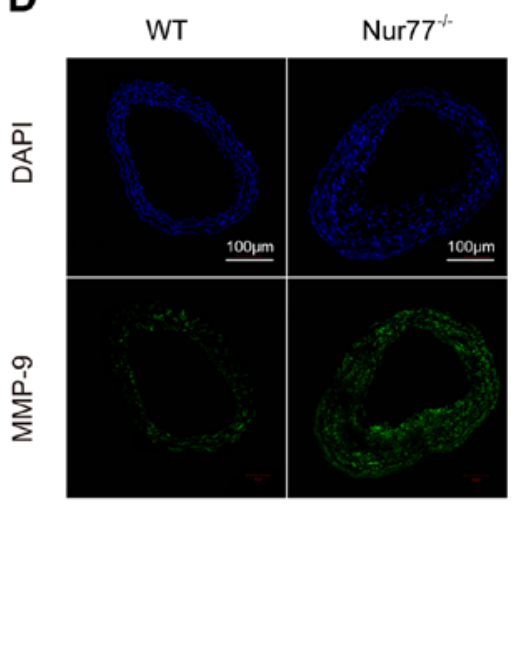

Figure 6. Nur77 deletion enhances vascular smooth muscle cells (VSMCs) proliferation and matrix metalloproteinase (MMP) expression. (A) Representative images of dual immunofluorescence staining of proliferating cell nuclear antigen (PCNA) and smooth muscle (SM)- $\alpha$-actin (scale bar, $100 \mu \mathrm{m}$ ) and quantification of the ratio of PCNA-positive area to $\alpha$-actin-positive area in wild-type (WT) and Nur77-deficent mice ( ${ }^{*} \mathrm{p}<0.05$, $\left.\mathrm{n}=6\right)$. (B and C) Representative images of (B) TUNEL staining (scale bar, $100 \mu \mathrm{m}$ ) and (C) quantification of the percentage of TUNEL-positive nuclei ( $\left.{ }^{*} \mathrm{p}<0.05, \mathrm{n}=6\right)$. (D and E) Representative images of (D) immunofluorescence staining of MMP-9 (scale bar, $100 \mu \mathrm{m})$ and (E) quantification of ratio of the MMP-9-positive area to intima area ( ${ }^{*} \mathrm{p}<0.05$, $\left.\mathrm{n}=6\right)$. Values represent the means \pm SEM.

area was significantly increased in the ligated carotid arteries from the Nur77-deficient mice compared to those from the WT mice $(73.17 \pm 1.89$ vs. $51.50 \pm 3.18 \%, \mathrm{p}<0.05)$ (Fig. 6A) On the contrary, there was no significant difference observed in the number of apoptotic vascular cells between the Nur77-deficient and WT mice (Fig. 6B and C). Moreover, immunofluorescence staining for MMP-9 revealed that the size of the MMP-9-positive area in the intima area was significantly increased in the ligated arteries from the Nur77-deficient mice compared to those from the WT mice $(25.79 \pm 2.69$ vs. $11.46 \pm 0.96 \%, \mathrm{p}<0.05$ ) (Fig. 6D and E). Taken together, these results suggest that Nur77 inhibits vascular remodeling induced by low shear stress in vivo by suppressing VSMC proliferation and MMP-9 production.

\section{Discussion}

Vascular remodeling is an important common pathophysiological process which is associated with a number of cardiovascular diseases. Moreover, vascular remodeling is a complex and heterogeneous process, which can be triggered and worsened by various cardiovascular risk factors, such as local hemodynamic dysfunction, hypertension and dyslipidemia. Also, shear stress, particularly low and oscillary shear stress, is known to play a crucial pathophysiological role in vascular remodeling-related cardiovascular complications, such as carotid IMT, coronary atherosclerosis, and even restenosis following angioplasty (1-4).

The most important finding of the current study is that, for the first time (to the best of our knowledge), we demonstrate that Nur77 deletion enhances vascular remodeling induced by low shear stress. The ligated carotid arteries from Nur77-deficient mice exhibited enhanced VSMC proliferation and MMP-9 production compared with those from WT mice. Furthermore, when the VSMCs were infected with Ad-GFP-Nur77, they exhibited a significantly decreased proliferation and migration following stimulation with PDGF. These findings demonstrate that Nur77 is an important negative regulator of the proliferation and migration of VSMCs and the development of low shear stress-induced vascular remodeling. Although some previous studies have examined the role of Nur77 in the biological behaviors of VSMCs and vascular remodeling $(15,16,25,26)$, this study, to the best of our knowledge, is the first to use Nur77-deficient mice to investigate the role which Nur77 plays in vascular remodeling induced by low shear stress, which may be the most common situation to mimic human vascular remodeling. This observation is consistent with the findings 
of previous studies (14-16) and strongly suggests that Nur77 inhibits the development of vascular remodeling.

Another important finding of this study was that the upregulation of Nur77 was ROS-dependent. We were able to demonstrate that Nur77 expression was increased in primary VSMCs following stimulation with PDGF or following vascular remodeling in vivo. In line with our data, it has been previously demonstrated that Nur77 expression is induced by diverse stimuli, including inflammatory stimuli, growth factors and cytokines, and it is also upregulated during atherosclerosis and vascular remodeling $(15,27,28)$. However, the underlying mechanisms through which Nur77 expression is regulated remain unclear. Previous research has suggested that oxidative stress is critical in vascular remodeling-related cardiovascular diseases (29). Accumulating data indicate that various risk factors can trigger vascular remodeling through the excessive generation of ROS (30). In this study, we observed increased levels of ROS both following stimulation of primary VSMCs in vitro with PDGF and following flow-induced vascular remodeling in vivo. Based on this result, we suggest that oxidative stress is the common reason for the upregulation of Nur77 during vascular remodeling. In the present study, we provided evidence that Nur77 acts as a sensor of oxidative stress and subsequently mediates the suppression of VSMC proliferation and migration following vascular remodeling induced by low shear stress.

From a therapeutic standpoint, our results have clinical implications for the treatment of vascular remodeling-related cardiovascular diseases. Modulating the expression or transcriptional activity of Nur77 represents a novel approach to inhibiting vascular remodeling. Pires et al demonstrated that the enhanced transcriptional activity of Nur77 by 6-mercaptopurine exerted protective effects against neointima formation (31). Moreover, it was demonstrated that $\alpha$-lipoic acid, which enhanced the cytoplasmic localization of Nur77 in VSMCs, triggered VSMC apoptosis and inhibited neointima formation in rat carotid arteries following balloon injury (32). Coupled with our new data, these findings suggest that Nur77 is a potential therapeutic target in the treatment of vascular remodeling-related cardiovascular diseases, such as atherosclerosis and restenosis following angioplasty.

In conclusion, in the present study, we demonstrate that Nur77 deletion enhances vascular remodeling induced by low shear stress. Our findings indicated that oxidative stress is the common trigger for the upregulation of Nur77 during vascular remodeling. Moreover, it can be noted that the increased ROS-dependent expression of Nur77 in turn inhibits the proliferation and migration of VSMCs and the development of vascular remodeling induced by low shear stress. Our study identified Nur77 as a potential novel therapeutic target for vascular remodeling. However, the identity of the downstream targets of Nur77 and the detailed mechanisms through which VSMCs function is regulated remain to be elucidated.

\section{Acknowledgements}

This study was supported by grant nos. 81070239, 30971185 and 81370399 from the National Natural Science Foundation; and grant no. 10JC1409400 from the Shanghai Municipal Natural Science Foundation.

\section{References}

1. Chatzizisis YS, Jonas M, Coskun AU, Beigel R, Stone BV, Maynard C, Gerrity RG, Daley W, Rogers C, Edelman ER, et al: Prediction of the localization of high-risk coronary atherosclerotic plaques on the basis of low endothelial shear stress: an intravascular ultrasound and histopathology natural history study. Circulation 117: 993-1002, 2008.

2. Carallo C, Irace C, Pujia A, De Franceschi MS, Crescenzo A, Motti C, Cortese C, Mattioli PL and Gnasso A: Evaluation of common carotid hemodynamic forces. Relations with wall thickening. Hypertension 34: 217-221, 1999.

3. Post MJ, Borst $\mathrm{C}$ and Kuntz RE: The relative importance of arterial remodeling compared with intimal hyperplasia in lumen renarrowing after balloon angioplasty. A study in the normal rabbit and the hypercholesterolemic Yucatan micropig. Circulation 89: 2816-2821, 1994.

4. Wentzel JJ, Gijsen FJ, Stergiopulos N, Serruys PW, Slager CJ and Krams R: Shear stress, vascular remodeling and neointimal formation. J Biomech 36: 681-688, 2003.

5. Qi YX, Jiang J, Jiang XH, Wang XD, Ji SY, Han Y, Long DK, Shen BR, Yan ZQ, Chien S, et al: PDGF-BB and TGF- $\beta 1$ on cross-talk between endothelial and smooth muscle cells in vascular remodeling induced by low shear stress. Proc Natl Acad Sci USA 108: 1908-1913, 2011.

6. Matlung HL, Bakker EN and VanBavel E: Shear stress, reactive oxygen species, and arterial structure and function. Antioxid Redox Signal 11: 1699-1709, 2009.

7. Lyle AN and Griendling KK: Modulation of vascular smooth muscle signaling by reactive oxygen species. Physiology (Bethesda) 21: 269-280, 2006.

8. Kratzer A, Buchebner M, Pfeifer T, Becker TM, Uray G, Miyazaki M, Miyazaki-Anzai S, Ebner B, Chandak PG, Kadam RS, et al: Synthetic LXR agonist attenuates plaque formation in apoE $\mathrm{E}^{-/-}$mice without inducing liver steatosis and hypertriglyceridemia. J Lipid Res 50: 312-326, 2009.

9. Pruthi D, McCurley A, Aronovitz M, Galayda C, Karumanchi SA and Jaffe IZ: Aldosterone promotes vascular remodeling by direct effects on smooth muscle cell mineralocorticoid receptors. Arterioscler Thromb Vasc Biol 34: 355-364, 2014.

10. Tobiasova Z, Zhang L, Yi T, Qin L, Manes TD, Kulkarni S, Lorber MI, Rodriguez FC, Choi JM, Tellides G, et al: Peroxisome proliferator-activated receptor- $\gamma$ agonists prevent in vivo remodeling of human artery induced by alloreactive $\mathrm{T}$ cells. Circulation 124: 196-205, 2011.

11. Hamers AA, Vos M, Rassam F, Marinković G, Kurakula K, van Gorp PJ, de Winther MP, Gijbels MJ, de Waard V and de Vries CJ: Bone marrow-specific deficiency of nuclear receptor Nur77 enhances atherosclerosis. Circ Res 110: 428-438, 2012.

12. Cheng Z, Völkers M, Din S, Avitabile D, Khan M, Gude N, Mohsin S, Bo T, Truffa S, Alvarez R, et al: Mitochondrial translocation of Nur77 mediates cardiomyocyte apoptosis. Eur Heart J 32: 2179-2188, 2011.

13. Wang RH, He JP, Su ML, Luo J, Xu M, Du XD, Chen HZ, Wang WJ, Wang Y, Zhang N, et al: The orphan receptor TR3 participates in angiotensin II-induced cardiac hypertrophy by controlling mTOR signalling. EMBO Mol Med 5: 137-148, 2013.

14. de Vries CJ, van Achterberg TA, Horrevoets AJ, ten Cate JW and Pannekoek H: Differential display identification of 40 genes with altered expression in activated human smooth muscle cells. Local expression in atherosclerotic lesions of smags, smooth muscle activation-specific genes. J Biol Chem 275: 23939-23947, 2000.

15. Arkenbout EK, de Waard V, van Bragt M, van Achterberg TA, Grimbergen JM, Pichon B, Pannekoek $H$ and de Vries CJ: Protective function of transcription factor TR3 orphan receptor in atherogenesis: decreased lesion formation in carotid artery ligation model in TR3 transgenic mice. Circulation 106: 1530-1535, 2002.

16. Bonta PI, Matlung HL, Vos M, Peters SL, Pannekoek H, Bakker EN and de Vries CJ: Nuclear receptor Nur77 inhibits vascular outward remodelling and reduces macrophage accumulation and matrix metalloproteinase levels. Cardiovasc Res 87: 561-568, 2010.

17. Sullivan CJ and Hoying JB: Flow-dependent remodeling in the carotid artery of fibroblast growth factor- 2 knockout mice. Arterioscler Thromb Vasc Biol 22: 1100-1105, 2002. 
18. Yang J, Li H, Chen YY, Wang XJ, Shi GY, Hu QS, Kang XL, $\mathrm{Lu}$ Y, Tang XM, Guo QS and Yi J: Anthraquinones sensitize tumor cells to arsenic cytotoxicity in vitro and in vivo via reactive oxygen species-mediated dual regulation of apoptosis. Free Radic Biol Med 37: 2027-2041, 2004.

19. Jovinge S, Hultgårdh-Nilsson A, Regnström $\mathrm{J}$ and Nilsson $\mathrm{J}$ : Tumor necrosis factor-alpha activates smooth muscle cell migration in culture and is expressed in the balloon-injured rat aorta. Arterioscler Thromb Vasc Biol 17: 490-497, 1997.

20. Raines EW: PDGF and cardiovascular disease. Cytokine Growth Factor Rev 15: 237-254, 2004

21. Kunsch C and Medford RM: Oxidative stress as a regulator of gene expression in the vasculature. Circ Res 85: 753-766, 1999.

22. Souza HP, Souza LC, Anastacio VM, Pereira AC, Junqueira ML, Krieger JE, da Luz PL, Augusto O and Laurindo FR: Vascular oxidant stress early after balloon injury: evidence for increased NAD $(\mathrm{P}) \mathrm{H}$ oxidoreductase activity. Free Radic Biol Med 28 1232-1242, 2000.

23. Szocs K, Lassègue B, Sorescu D, Hilenski LL, Valppu L, Couse TL, Wilcox JN, Quinn MT, Lambeth JD and Griendling KK Upregulation of Nox-based nad(p)h oxidases in restenosis after carotid injury. Arterioscler Thromb Vasc Biol 22: 21-27, 2002.

24. de Graaf R, Tintu A, Stassen F, Kloppenburg G, Bruggeman C and Rouwet $\mathrm{E}$ : $\mathrm{N}$-acetylcysteine prevents neointima formation in experimental venous bypass grafts. Br J Surg 96: 941-950, 2009

25. de Waard V, Arkenbout EK, Vos M, Mocking AI, Niessen HW, Stooker W, de Mol BA, Quax PH, Bakker EN and VanBavel E: TR3 nuclear orphan receptor prevents cyclic stretch-induced proliferation of venous smooth muscle cells. Am J Pathol 168 2027-2035, 2006.
26. Wang L, Gong F, Dong X, Zhou W and Zeng Q: Regulation of vascular smooth muscle cell proliferation by nuclear orphan receptor nur77. Mol Cell Biochem 341: 159-166, 2010.

27. Shao Q, Shen LH, Hu LH, Pu J, Qi MY, Li WQ, Tian FJ, Jing Q and He B: Nuclear receptor Nur77 suppresses inflammatory response dependent on COX-2 in macrophages induced by oxLDL. J Mol Cell Cardiol 49: 304-311, 2010.

28. Pei L, Castrillo A, Chen M, Hoffmann A and Tontonoz P: Induction of NR4A orphan nuclear receptor expression in macrophages in response to inflammatory stimuli. J Biol Chem 280: 29256-29262, 2005.

29. Higashi Y, Noma K, Yoshizumi M and Kihara Y: Endothelial function and oxidative stress in cardiovascular diseases. Circ J 73: 411-418, 2009.

30. Yung LM, Leung FP, Yao X, Chen ZY and Huang Y: Reactive oxygen species in vascular wall. Cardiovase Hematol Disord Drug Targets 6: 1-19, 2006.

31. Pires NM, Pols TW, de Vries MR, van Tiel CM, Bonta PI, Vos M, Arkenbout EK, Pannekoek H, Jukema JW, Quax PH and de Vries CJ: Activation of nuclear receptor Nur77 by 6-mercaptopurine protects against neointima formation. Circulation 115: 493-500, 2007.

32. Kim HJ, Kim JY, Lee SJ, Kim HJ, Oh CJ, Choi YK, Lee HJ, Do JY, Kim SY, Kwon TK, et al: $\alpha$-Lipoic acid prevents neointimal hyperplasia via induction of p38 mitogen-activated protein kinase/Nur77-mediated apoptosis of vascular smooth muscle cells and accelerates postinjury reendothelialization. Arterioscler Thromb Vasc Biol 30: 2164-2172, 2010. 